\title{
Crecimiento Económico y Gasto Público. Estimación Departamental
}

\section{Mary Ruth Fonseca Becerra}

Universidad Santo Tomas

Facultad de Economía

Bogotá D.C., Colombia

2015 


\title{
Crecimiento Económico y Gasto Público. Estimación Departamental
}

\author{
Mary Ruth Fonseca Becerra \\ Tesis presentada como requisito parcial para optar el título de: \\ Magister en Ciencias Económicas
}

Director:

Gustavo Junca Rodríguez

Universidad Santo Tomas

Facultad de Economía

Bogotá D.C., Colombia

2015 


\section{TABLA DE CONTENIDO}

$\mathrm{Pg}$

INTRODUCCIÓN

6

1. PLANTEAMIENTOS TEÓRICOS..............................................9

Familias.................................................................... 11

Empresas ... .................................................... 13

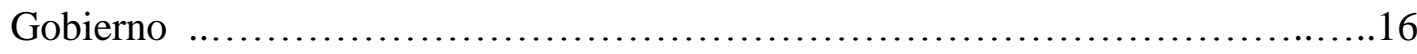

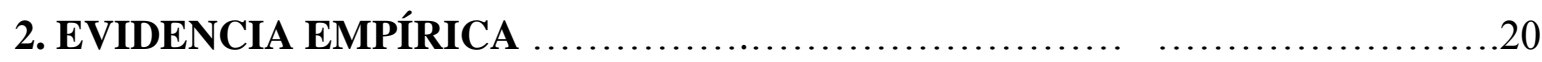

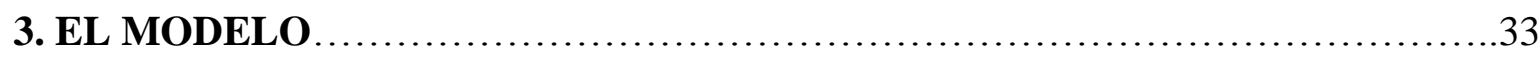

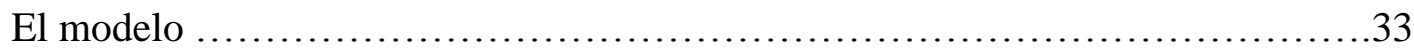



Metodología de estimación utilizada......................................34

Estimación del Modelo........................................................

4. RESULTADOS, CONCLUSIONES Y RECOMENDACIONES...............38

Resultados............................................................. 38

Conclusiones y Recomendaciones........................................43

LISTA DE REFERENCIAS...............................................46 


\section{INTRODUCCIÓN}

La actual organización territorial del Estado colombiano fue establecida en la Constitución de 1991. De acuerdo con el artículo $1^{\circ}$ de la Constitución Política, Colombia es un Estado unitario pero con cierto grado de autonomía en materia política, fiscal y administrativa autonomía de sus entidades territoriales, entendidas estas como los departamentos, los distritos, los municipios y los territorios indígenas.

En desarrollo del precepto constitucional si bien es cierto se han concedido competencias a las entidades territoriales estas son muy reducidas; ya que nación se reserva la competencia de regulación en todos los sectores mediante la expedición de leyes y decretos reglamentarios. Las competencias territoriales tienen que ver con algunas normas que pueden dictar a través de sus asambleas y consejos pero con sujeción a las disposiciones legales que dicta la nación. El ámbito donde gozan de mayor autonomía es en materia de ordenamiento territorial, y específicamente en lo relativo a la regulación de los usos del suelo; seguido de algunas competencias relativas a la provisión de servicios de salud, de educación preescolar, primaria, secundaria y media, y los servicios públicos domiciliarios de agua potable y saneamiento básico, con el fin de garantizar la prestación y la ampliación de coberturas, con énfasis en la población pobre.

\footnotetext{
${ }^{1}$ Artículo 356 de la Constitución Política de 1991. Salvo lo dispuesto por la Constitución, la ley, a iniciativa del Gobierno, fijará los servicios a cargo de la Nación y de los Departamentos, Distritos, y Municipios. Para efecto de atender los servicios a cargo de éstos y a proveer los recursos para financiar adecuadamente su prestación, se crea el Sistema General de Participaciones de los Departamentos, Distritos y Municipios(...).
} 
En este contexto resulta importante preguntarse cuál es el grado de importancia de cada una de estas divisiones político administrativas y cuál su impacto individual sobre el crecimiento económico y el mejoramiento de la calidad de vida de la población, ante todo considerando que entre la nación y los municipios ocupan cerca del $80 \%$ del total de la inversión de los recursos en los diferentes sectores; lo cual deja a los departamentos con apenas una participación cercana al 20\% concentrada en los sectores de educación y salud.

Este trabajo pretende abordar el cuestionamiento planteado desde la perspectiva del gasto público regional. En Colombia los estudios del impacto del gasto público sobre el crecimiento demuestran que éste es un motor de crecimiento, no obstante la evidencia a nivel regional es escasa, por tanto resulta importante preguntar ¿cuál ha sido el impacto del gasto público productivo sobre el crecimiento regional?, con el fin de determinar si la dinámica que ha presentado el gasto productivo a nivel agregado se cumple a nivel regional.

Para responder al interrogante planteado, el documento se ha divido en cinco secciones principales, una de las cuales es esta introducción. La sección II presenta el modelo teórico y sus implicaciones; de manera sencilla y entendible se desarrolla el crecimiento endógeno, sobre la base de la alternativa de interpretación de la tecnología AK ofrecida por Barro en 1990, que se caracteriza por la introducción de factores productivos de provisión pública en la función de producción. 
La sección III relaciona los resultados de la evidencia empírica en el país de los que se puede concluir que a pesar de algunos avances importantes en materia de descentralización y desarrollo regional aún persisten las desigualdades regionales en términos de crecimiento económico y la calidad de vida; la sección IV presenta la descripción de las variables y datos empleados y el modelo econométrico estimado mediante la metodología de datos panel para poner a prueba el modelo teórico y estimar sus parámetros; cabe destacar la dificultad que se presenta debido a la limitación y deficiencias que presenta la producción de estadísticas regionales de calidad y consistencia metodológica.

La sección V presenta los resultados y conclusiones, las que permiten colegir que el gasto público regional si impacta los resultados de crecimiento económico, no obstante persisten las condiciones de desigualdad e inequidad entre las regiones; por tanto sigue siendo un reto de la sociedad y de los hacedores de política el diseño de medidas que reduzcan las desigualdades, potencien el desarrollo económico equilibrado de las regiones y permitan superar los resultados de los indicadores de pobreza. 


\section{PLANTEAMIENTOS TEÓRICOS}

Los economistas profesionales y los responsables de la política económica derivan de la teoría pautas que sirven de sustento a la política y guían las acciones del Estado. Estás últimas le permiten al gobierno utilizar variables para compensar efectos que se consideran indeseados o para provocar comportamientos en otras variables que coadyuven a alcanzar los objetivos macroeconómicos básicos: crecimiento, empleo e inflación.

Entre la gamma de instrumentos de la que puede disponer el gobierno, la política fiscal juega un papel importante y, a la vez, suscita grandes debates. Uno de los aspectos que inquietan y generan controversia teórica y práctica es el relativo al rol que desempeña el Estado, a través de los componentes productivos del gasto público, como determinante del crecimiento económico, puesto que por un lado el de gasto público en capital humano e infraestructura física puede estar positivamente relacionado con la tasa de crecimiento económico, pero por otro la tendencia a volverse excesivo genera cargas para financiarlo, lo cual afecta de manera negativa el crecimiento.

El capítulo se dedica a exponer de manera sencilla y entendible los conceptos teóricos alrededor de la teoría de crecimiento endógeno, sobre la base de la alternativa de 
interpretación de la tecnología AK ofrecida por Barro en 1990, caracterizada por la introducción de factores productivos de provisión pública en la función de producción ${ }^{2}$.

Los modelos de crecimiento endógeno muestran, a diferencia de los planteamientos neoclásicos, una tasa de crecimiento a largo plazo positiva sin necesidad de recurrir a ninguna variable exógena determinada fuera del modelo. La corriente de economistas que propone este tipo de modelos, afirma que los modelos neoclásicos no proporcionan conclusiones satisfactorias en la explicación del fenómeno del crecimiento, básicamente por tres motivos:

1. La imposibilidad de capturar, dentro del modelo, el efecto a largo plazo sobre la tasa de crecimiento de fenómenos económicos como el esfuerzo inversor, los procesos de investigación y desarrollo (I+D), el gasto público o la fiscalidad.

2. Los modelos neoclásicos no permiten explicar de forma satisfactoria las causas por las cuales las tasas de crecimiento son tan diferentes entre los países.

3. No resulta explicado de una forma convincente por qué no se producen grandes movimientos de capital desde los países ricos hacia los pobres, en los que la productividad marginal del capital es mayor, y que de acuerdo con las hipótesis neoclásicas deberían ser mayores.

\footnotetext{
$2{ }^{2}$ EL desarrollo del modelo teórico se toma del libro de Barro R. \& X. Sala-i-Martin, (1995), Economic Growth, McGraw-Hill, en su versión en español.
} 
En estos modelos, al hacer endógenos los factores que determinan el crecimiento de las economías, sí existe la posibilidad de afectar al crecimiento a largo plazo. Así, por lo que se refiere al ámbito de la política fiscal, los modelos de crecimiento endógeno consideran que las variables fiscales pueden tener un efecto importante sobre el crecimiento.

Dentro de la literatura de crecimiento endógeno, Barro(1990) aporta una interpretación alternativa de la tecnología AK propuesta formalmente por Rebelo(1991), en la cual considera que la producción depende del capital privado y de un factor de producción, que el mismo Barro califica como de un bien privado cuya provisión está a cargo del sector público.

Bajo un contexto de mercados competitivos se determina, en primera medida, el comportamiento de las familias y empresas, para después considerar la actuación del sector público.

\section{Las Familias}

Las familias toman sus decisiones de consumo y ahorro maximizando su utilidad intertemporal sujeta a una restricción presupuestal. Las preferencias en cada período vienen representadas por una función de utilidad de horizonte infinito dependiente exclusivamente del consumo $c_{t}$, de la forma 
$U=\int_{0}^{\infty} e^{-(\rho-n) t} \cdot\left[\frac{c^{(1-\theta)}-1}{(1-\theta)}\right] d t$

donde $\rho$ es una constante que representa la tasa de descuento, $c$ es el consumo per cápita en el momento t, $n$ es la tasa constante de crecimiento de la población ${ }^{3}$ y $\theta$ es la inversa de la elasticidad de substitución intertemporal, que también es constante y refleja el mayor o menor interés de los individuos por suavizar su consumo a través del tiempo.

Las familias enfrentan una restricción presupuestaria dada por

$a=(r-n) \cdot a+w-c$

donde $a$ son los activos per cápita, $r$ es la tasa de interés, $w$ es la tasa de salario al que las familias alquilan su trabajo, y $n$ es la tasa de crecimiento de la población.

En este contexto los agentes individuales toman las variables presupuestarias como dadas, es decir, cuando resuelven su problema de optimización no son concientes del efecto que tienen sus decisiones de consumo e inversión sobre las mismas, en este orden de ideas el Hamiltoniano será

\footnotetext{
${ }^{3}$ Se supone que la tasa de crecimiento de la población es constante e igual a $n$, de manera que $L / L=n$, por lo que la población en el momento $t$ es igual a $L_{t}=(0) e^{n t}$. Si normalizamos la población inicial, $L(0)=1$, tenemos que la población en el instante $t$ viene dada por $L_{t}=e^{n t}$.
} 


$$
H(\cdot)=e^{-(\rho-n) t}\left(\frac{c^{(1-\theta)}-1}{1-\theta}\right)+v[w+(r-n) a-c]
$$

donde $v$ es el precio implícito de los activos $a$.

Las condiciones de primer orden son las siguientes:

$$
\begin{aligned}
& H_{c}=0 \leftrightarrow e^{-(\rho-n) t} c^{-\theta}=v \\
& H_{b}=-\dot{v} \leftrightarrow-\dot{v}=v(r-n)
\end{aligned}
$$

y la condición de transversalidad

$$
\lim _{t \rightarrow \infty} a_{t} v_{t}=0
$$

De las condiciones anteriores, se halla la condición que debe cumplir el crecimiento del consumo

$$
\gamma_{c}=\frac{\dot{c}}{c}=\frac{1}{\theta}(r-\rho)
$$

\section{Las Empresas}

La tecnología disponible para las empresas esta descrita por una función de producción de tipo Cobb - Douglas, que en particular para este tipo de modelos se caracteriza por que es 
lineal en el único factor de producción: el capital. La función de producción presenta rendimientos constantes de escala y rendimientos decrecientes en cada uno de los factores ${ }^{4}$. $y=f(k)=A k$

siendo $A>0$, una constante exógena que representa el nivel de tecnología y afecta la tasa de crecimiento per cápita de largo plazo y $k$ el capital per cápita agregado, definido de una manera amplia, en la cual se incluyen el capital humano y el capital físico.

Las condiciones para maximizar la ganancia requieren que el producto marginal del capital sea igual al precio de alquiler del capital, $R=r+\delta$, y dado que el producto marginal del capital es la constante $A$, entonces obtenemos $r=A-\delta$.

El producto marginal del trabajo es igual a cero, por tanto la tasa de salario será igual a cero ${ }^{5}$.

En el modelo propuesto por Barro(1990), se trabaja bajo el contexto de una economía cerrada, lo cual implica que la condición de equilibrio del mercado financiero requiere que el único activo con oferta neta positiva sea al capital, por lo que se cumple que $a=k$ y si

4 La función $\mathrm{AK}$, no cumple todas las condiciones neoclásicas: Exhibe rendimientos positivos pero no decrecientes del capital dado que $\frac{\partial Y}{\partial K}=A$ y $\frac{\partial^{2} Y}{\partial K^{2}}=0 ;$ tampoco satisface las condiciones de Inada, dado que el producto marginal del capital es siempre igual a $A$, por lo que no se aproxima a cero cuando $K$ se aproxima a infinito y no se aproxima a infinito cuando $K$ se aproxima a cero $\left(\lim _{k \rightarrow \infty} F^{\prime}(K)=A \neq 0\right.$ y $\left.\lim _{k \rightarrow 0} F^{\prime}(K)\right)=A \neq \infty$.

${ }^{5}$ Las familias se mantienen constantes y normalizados a la unidad. 
$r=A-\delta \quad$ y $w=o$, condiciones que sustituidas en las ecuaciones (2), (6) y (7) dan como resultado

$$
\begin{gathered}
\dot{k}=(A-\delta-n) \cdot k-c \\
\gamma_{c}=\frac{1}{\theta} \cdot(A-\delta-\rho) \\
\lim _{t \rightarrow \infty}\left\{k(t) \cdot e^{-(A-\delta-n) \cdot t}\right\}=0
\end{gathered}
$$

De los resultados obtenidos es importante resaltar que la función de producción garantiza el crecimiento de $c$, y a su vez asegura que la utilidad sea acotada, para lo cual es necesario el cumplimiento de algunas condiciones:

$$
A>\rho+\delta>[(1-\theta) / \theta] \cdot(A-\delta-\rho)+n+\delta .
$$

Por definición de estado estacionario la tasa de crecimiento del consumo, del capital y de la producción per cápita son idénticas y vienen dadas por

$$
\gamma_{k}=\gamma_{c}=\gamma_{y}=\gamma=\frac{1}{\theta}[A-\delta-\rho]
$$

De la ecuación anterior, tenemos que valores pequeños de $\rho$ y $\theta$ incrementan el deseo de ahorro lo que genera incremento en la tasa de crecimiento del producto per cápita. Un mejoramiento en el nivel de tecnología $A$, incrementa el producto medio y marginal del capital incrementa la tasa de crecimiento. 


\section{El Gobierno}

El objetivo de Barro (1990), es mostrar que varias actividades del gobierno pueden generar efectos sobre el coeficiente $A$, y por tanto, sobre la tasa de crecimiento. El principal supuesto es considerar el gasto público como deseable y productivo, para lo cual se introducirá como un argumento positivo en la función de producción.

El bien público $g$, se define como un bien privado, rival y excluible que será provisto por el sector público y que tiene la característica de ser un flujo productivo, en vez de un bien acumulable. Las acciones del Estado incluyen actividades como la provisión de infraestructura, la protección de los derechos de propiedad, y la tasación de la actividad económica.

Con la introducción de la actividad pública del Estado la tecnología disponible para las empresas esta descrita por una función de producción que utiliza inputs de capital privado $k$ y un factor de provisión pública y gratuito para las empresas $g$, la función conserva las propiedades originales de rendimientos constantes a escala, pero decrecientes en cada uno de los factores, de la forma

$$
y=A k^{1-\alpha} g^{\alpha}
$$

Nuevamente se asume que $L$, la fuerza laboral es constante y normalizada a la unidad. 
La función de producción implica que los servicios públicos son complementarios con los inputs privados en el sentido que un incremento en $g$ incrementa el producto marginal de $k$

En el escenario definido por Barro (1990), el sector público mantiene sus cuentas equilibradas de forma el saldo presupuestario en todo momento es cero ${ }^{6}$. La restricción presupuestaria del sector público se expresa como

$g=\tau y=\tau A k^{\alpha} g^{1-\alpha}$

El problema de maximización de las empresas cambia, puesto que ahora maximizarán su beneficio neto de impuestos

$\pi_{\text {neto }}=(1-\tau) A k^{\alpha} g^{1-\alpha}-R k$

donde $R$ es el precio de alquiler del capital, que es igual a $R=r+\delta$.

La maximización del beneficio implica que el producto marginal neto del capital sea igual a la tasa de alquiler

\footnotetext{
${ }^{6}$ En la práctica los gobiernos incurren en déficit o superávit fiscales, no obstante, el modelo se trabaja en un escenario de largo plazo en el cual el presupuesto debe estar mas o menos equilibrado (Sala - i - Martín) (1994).
} 
$(1-\tau) A \alpha k^{\alpha-1} g^{1-\alpha}=r+\delta$.

substituyendo esta expresión en la ecuación de optimización de las familias consumidoras (7), obtenemos que la tasa de crecimiento del consumo viene dada por

$$
\frac{\dot{c}}{c} \equiv \gamma_{c}=\left((1-\tau) A \alpha\left(\frac{g}{k}\right)^{1-\alpha}-(\delta+\rho)\right)
$$

De la restricción presupuestaria del gobierno, ecuación (15), expresamos el tipo impositivo como función de $g / k$, de modo que

$$
\tau=\frac{g}{y}=\frac{g}{A k^{\alpha} g^{1-\alpha}}=\frac{(g / k)^{\alpha}}{A} .
$$

despejando $g / k$, obtenemos que

$g / k=(\tau A)^{1 / \alpha}$.

donde a través de la sustitución de (20) en $\frac{\dot{c}}{c}$, obtenemos la nueva expresión de crecimiento del consumo, donde la actividad pública afecta las tasas de crecimiento de largo plazo.

$$
\frac{\dot{c}}{c}=\gamma_{c}=\frac{1}{\theta}\left((1-\tau) \alpha A^{1 / \alpha} \tau^{1-\alpha / \alpha}-(\delta+\rho)\right) .
$$

Los efectos de la actividad del gobierno vienen dados por dos vías. Por un lado, $1-\tau$ representa el efecto negativo de la carga tributaria sobre el producto marginal del capital 
después de impuestos y el término $\tau^{(1-\alpha) / \alpha}$, representa el efecto positivo de la acción estatal, logrado por la provisión de servicios públicos, sobre el producto marginal.

La expresión (21), conlleva una disyuntiva sobre la actividad pública. Los efectos positivos y negativos derivados de la existencia de un Estado que provee bienes y servicios productivos implica la elección de un nivel óptimo de actividad pública que permita equilibrar los efectos de la política pública sobre la actividad privada ${ }^{7}$.

El óptimo del tamaño del Estado, en consecuencia, se obtendrá donde $\tau$ haga máximo el valor de $\gamma$. El máximo de la función se encuentra igualando a cero la derivada de la tasa de crecimiento con respecto a $\tau$, encontrando que

$$
\tau^{*}=\frac{G}{Y}=1-\alpha .
$$

La expresión gráfica de la ecuación (21), permite ver que para valores bajos de $\tau$, el efecto positivo de la acción pública sobre el producto marginal del capital es dominante y, por tanto, $\gamma$ se incrementa con $\tau$. El comportamiento ascendente continúa hasta alcanzar un pico donde el tamaño del Estado llega al óptimo, y a partir del cual el efecto de la acción pública,

${ }^{7}$ Sala-i-Martín (1994), explica la relación existente entre el tamaño del Estado y la tasa de crecimiento de la siguiente manera. Si $\tau$ es cero, la productividad marginal del capital después de impuestos también vale cero, por lo que la tasa de crecimiento es negativa $\gamma^{*}=(1 / \theta)(-\rho-\delta)$. Esto se debe a que cuando $\tau$ es cero, el Estado no puede proporcionar bienes públicos. Cuando no existen bienes públicos, el rendimiento de la inversión privada es cero. En el otro extremo cuando $\tau$ vale 1, el Estado proporciona una cantidad enorme de bienes públicos, que hacen que el capital privado sea muy productivo. Ahora bien, estos bienes siempre se tienen que financiar y ello conlleva distorsiones que reducen los incentivos a la inversión y el crecimiento. 
representado por la distorsión adversa que introducen los impuestos, empieza a dominar y $\gamma$ comienza a declinar.



Tal y como lo concluyen Posada y Escobar(2003), el modelo teórico de Barro expresa que el gasto público puede ser productivo pero que más allá de un cierto nivel es un freno a la producción: si el gasto público es inferior o superior a cierta magnitud la tasa de crecimiento de la economía será inferior a la máxima, y si el gasto público es tal magnitud se alcanza la tasa máxima de crecimiento. 


\section{EVIDENCIA EMPIRICA}

La diferente gama de estudios sobre los efectos del gasto público en el contexto macroeconómico en Colombia, llevan a valorar la gestión del Estado a partir del análisis de la disyuntiva que genera el constante incremento del gasto público de los últimos años y su efecto sobre el comportamiento de los principales indicadores sectoriales, junto con el deterioro continuo en las finanzas del Estado.

No obstante, resulta un reto de mayores magnitudes el establecer si los grandes esfuerzos de financiación del gobierno se han visto reflejados en mejoras en la calidad de vida de la población.

Satisfacer estos cuestionamientos requiere de respuestas a preguntas como las siguientes, que últimas llevan a definir un tamaño óptimo del Estado: ¿Se podría afirmar que en incremento del gasto en defensa y seguridad que pasó de $2.1 \%$ a $4.03 \%$ del PIB, entre 1994 y 2002, así como el gasto social que pasó de $11 \%$ del PIB en 1994 a $14.9 \%$ en 2004, y se mantuvo en niveles históricamente altos durante el período 1998-2004 (gráfico 1) ${ }^{8}$, aún en condiciones de ajuste estructural, y en medio de la más grave recesión económica de los últimos 70 años,

\footnotetext{
${ }^{8}$ Fuente de datos: Documento Conpes № 329428 de Junio de 2004
} 
han impactado de manera positiva y significativa los niveles de desarrollo económico de la Nación?.

\section{Gráfico Nº 1}

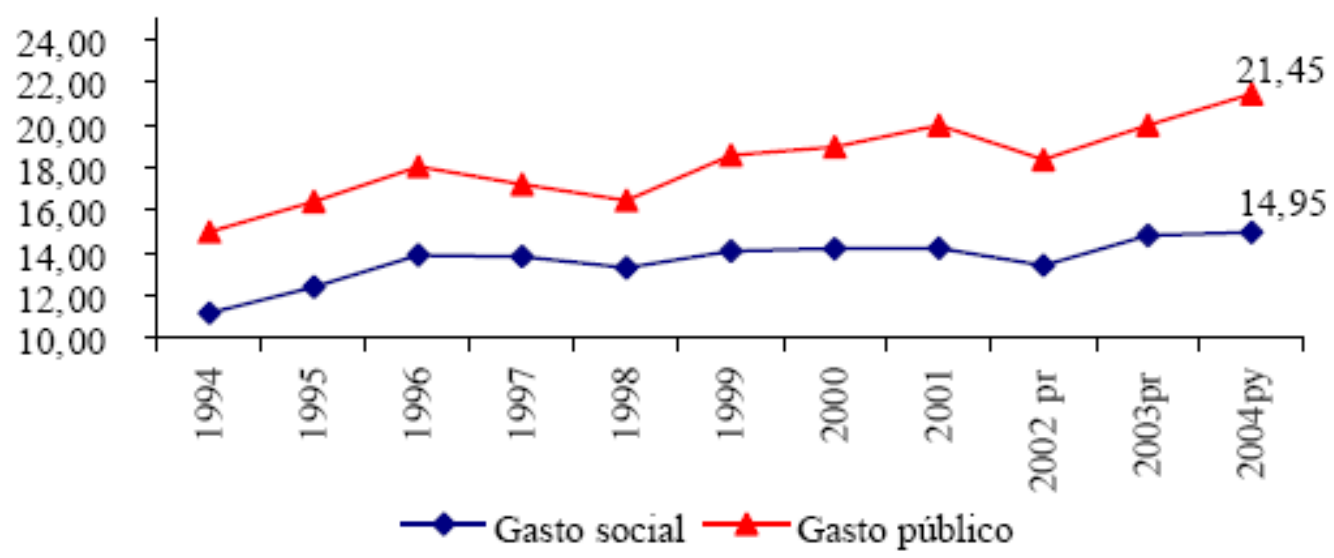

Tal como se plasma en el Documento Conpes 3294, "hasta el momento se desconoce si el uso de los recursos de transferencias por parte de los departamentos y municipios está afectando positivamente, la salud, la nutrición o la escolaridad de la población, y en que medida. Esto se debe primordialmente, a la precariedad de la información disponible, al débil control social en las regiones y a la ausencia de evaluaciones de impacto de estos recursos sobre los beneficiarios y las regiones.

En conclusión, a pesar de la magnitud de los recursos públicos invertidos durante la última década, dada la insuficiencia de evaluaciones del gasto y de los programas públicos, hasta el momento se desconocen los efectos reales de estas inversiones. Ello impide determinar con certeza si la orientación de las políticas ha sido la más adecuada en términos de eficiencia, si los programas llegan a la población objetivo y si responden a sus necesidades, 
si es la forma menos costosa de realizar las intervenciones; o si tienen los niveles de calidad requeridos entre otros aspectos.

Dada la magnitud creciente del gasto y la desaceleración prolongada del ritmo de crecimiento, y la incertidumbre frente a la efectividad de las políticas públicas se hace imperativo avanzar en los asuntos referidos a los motores y frenos del crecimiento, y a sus perspectivas futuras ${ }^{9}$.

Avanzando en este tipo de cuestiones, en este capítulo se recopilarán una serie de resultados, tanto a nivel agregado como regional, que permiten ubicar el papel del gasto público, especialmente en sus componentes productivos, sobre la tasa de crecimiento del producto, mostrando resultados interesantes que sitúan la efectividad de la política fiscal en la identificación de un nivel óptimo y en el tipo del gasto que se ejecuta.

Por un lado, trabajos como el de Posada y Escobar ( 2002), por medio de la estimación econométrica del modelo de gasto público y crecimiento econmico de Barro, encontraron que el comportamiento alcista del gasto público, que desde los años 80 ya era excesivo para hacer máxima la tasa de crecimiento del producto, contribuyó a la reducción de la tasa de

\footnotetext{
${ }^{9}$ Posada y Gómez (2002). De acuerdo con Bleaney y Nishiyama (2002) la evidencia empírica acumulada hasta el presente permite considerar la existencia de un modelo económico de "patrón”, de cuya estimación se estableció que existen 14 variables significativas para explicar la tasa de crecimiento anual del PIB, a saber: Ingreso per cápita inicial $Y$, apertura, apertura por $Y$, expectativa de vida al nacer, escolaridad masculina post - primaria, calidad institucional, índice de democracia, , ahorro del gobierno central, participación de las exportaciones de productos primarios en el PIB, crecimiento de los términos de intercambio, clima tropical, diferencia en los crecimientos de la población económicamente activa y total.
} 
crecimiento de la economía. De otra parte, trabajos como el de Fabio Sánchez (1993), en el que se estudio el impacto del capital público sobre la productividad del capital industrial y privado, sobre la tasa de inversión privada y sobre el crecimiento económico, concluye que el capital público y fundamentalmente la llamada infraestructura núcleo tiene un impacto positivo sobre la productividad, la tasa de inversión y por consiguiente sobre el crecimiento económico.

Posteriormente, Posada y Gómez (2002), presentan un modelo de crecimiento económico, en el que se analiza particularmente el gasto público en los rubros de capital humano (salud y educación) e infraestructura física, en el que concluyen que este tipo de gasto es importante para impulsar el crecimiento, pero también genera una pérdida de bienestar derivada de su fuente de financiación: impuesto a la renta. Las estimaciones arrojan como nivel de gasto público óptimo para la sociedad el 20\% del PIB: $15.1 \%$ en capital humano, que se asimila a gasto en educación y salud, y $4.9 \%$ en infraestructura.

En términos generales, los estudios del impacto del gasto público sobre el crecimiento económico permiten concluir que es necesario que el gasto público se sitúe y mantenga en el nivel óptimo para que propicie efectos positivos sobre la tasa de crecimiento, al igual es importante destinar recursos a rubros de gasto público en capital humano (salud y educación) e infraestructura, los cuales contribuyen a situar la economía en círculos virtuosos de crecimiento. 
Los asuntos relacionados con el crecimiento económico regional y sus determinantes presenta dificultades, debido tal vez, a las deficiencias que presentan los datos regionales: No existen series consolidadas para todas las unidades territoriales para un buen número de años, existen diferencias en las metodologías de recolección de datos. Sin embargo, enfrentando las limitaciones existentes, se han desarrollado estudios encaminados primordialmente a comprobar la hipótesis de convergencia, con resultados interesantes, que permiten evidenciar las características de los patrones de crecimiento regional.

Para el caso colombiano, se reseñarán algunos trabajos que ilustran las principales características del crecimiento regional, y además permiten apreciar la importancia que ha tenido el gasto público productivo, representado por la inversión en educación, salud e infraestructura; como motor de crecimiento regional.

Meisel y Galvis (2000), evalúan la evolución de las disparidades económicas entre las principales ciudades colombianas y los determinantes de su crecimiento para el período 1973-1998, con una muestra de 20 ciudades bajo las consideraciones teóricas del modelo de Robert Barro y Xavier Sala- I-Martín (1995), encontrando que en Colombia se ha presentado un proceso de polarización urbana, en el que las disparidades entre la ciudades han crecido notablemente.

Las estimaciones muestran como principales fuentes de crecimiento de las ciudades en el período 1973-1998: el capital humano, medido en términos de la cobertura de la educación 
secundaria, del porcentaje de personas que ha asistido a la universidad y del promedio ICFES; la infraestructura física, medida por el porcentaje de hogares con acceso a los servicios públicos básicos y líneas por cada mil habitantes; las instituciones, medidas por el recaudo tributario predial e industria y comercio; la tasa de homicidios como indicador de la violencia; la tasa de fecundidad y el crecimiento de la población, al igual que algunas variables relacionadas con la localización y la geografía física.

El análisis encuentra que se está presentando un proceso de concentración de la riqueza entre las ciudades estudiadas, concentración que se ha acentuado a partir de la década de los 90, y que ha generado que las desigualdades persistan y se amplíen.

El estudio llegó a la conclusión que las variables que contribuyen a la explicación de la tasa y el nivel de crecimiento del PIB per cápita urbano son el capital humano y la infraestructura. También resultaron significativas el tamaño de la población en el 1973, la tasa de crecimiento poblacional, y la tasa de fertilidad; mientras que el desempeño de las instituciones y las variables geográficas no resultaron significativas.

En conclusión, los resultados muestran que en las últimas tres décadas Colombia ha venido experimentando un proceso de polarización urbana, el cual se refleja en la creciente disparidad del PIB de las principales ciudades. No hay evidencia de procesos de convergencia $\beta$ no condicional, ni convergencia $\sigma$. Es importante resaltar que las variables geográficas usadas en la literatura empírica sobre crecimiento, inspiradas en Jeffrey Sachs no resultaron 
relevantes para entender el crecimiento de las ciudades colombianas, tal vez por las dificultades para ser capturadas por herramientas econométricas sencillas, o porque sólo puede ser entendidas desde una perspectiva histórica.

El resultado mas importante es haber demostrado que las variables que mayor efecto tienen sobre la tasa de crecimiento del PIB per cápita de las ciudades y de su nivel, son el capital humano y la dotación de infraestructura física y que por tanto cualquier política encaminada a lograr una convergencia entre los niveles de ingreso per cápita de las ciudades y regiones colombianas tendrá necesariamente, un fuerte componentes de inversión en educación e infraestructura, para poner a las regiones pobres en condiciones que les permitan competir con las más ricas en pie de igualdad.

Por su parte Cárdenas, Pontón y Trujillo (1993), analizan sistemáticamente los datos departamentales colombianos para el período 1950 -1989, con el propósito de determinar si ha habido a lo largo de la posguerra una tendencia hacia la convergencia en los ingresos por habitante entre los diferentes departamentos del país, cuáles han sido los determinantes fundamentales de la migración interdepartamental en el país y, por último, qué papel han jugado los flujos migratorios desde el punto de vista de la convergencia entre departamentos.

Las conclusiones de trabajo indican que Colombia ha sido un caso exitoso de convergencia regional, encontrando una tasa de convergencia tipo $\beta$ del $4 \%$ por año, lo cual quiere decir que el país acorta los diferenciales en el ingreso per cápita entre departamentos a una 
velocidad que duplica la estimada en EEUU, Europa y Japón. También existe una tendencia definida hacia la convergencia tipo $\sigma$ entre departamentos.

El estudio encuentra una fuerte relación positiva entre el ingreso per cápita y la inmigración hacia un departamento, lo que verifica que las oportunidades económicas son el principal determinante de los flujos migratorios, también se confirma que a mayor violencia, mayor es la expulsión de población hacia otras áreas del país, igual los migrantes evitan departamentos con inestabilidad política, también se encuentra que los flujos migratorios no contribuyen significativamente al proceso de convergencia. Los departamentos que invierten más en educación crecen con mayor rapidez, independientemente de su situación inicial.

Otro estudio realizado por Cárdenas (1993) para el período 1950-1990, en el cual el autor realiza algunas pruebas de los resultados obtenidos en la literatura internacional a partir de un conjunto de datos recopilados en las cuentas regionales colombianas, para investigar hasta qué punto existen fuerzas hacia la convergencia del ingreso por habitante en las diferentes regiones del país y explorar sobre las características del desarrollo económico de Colombia desde una perspectiva regional, con énfasis en el análisis cuantitativo.

Con interrogantes muy similares al documento anteriormente citado, a saber: ¿ha habido alguna convergencia en el ingreso per cápita de las diferentes regiones del país?, ¿cuál ha sido el papel de la migración en ese contexto? y i por qué el crecimiento económico de algunas regiones ha sido mayor que el de otras? 
Nuevamente encuentra que las variables representativas del capital humano, como la tasa de cobertura de la educación primaria, la tasa de analfabetismo, la cobertura de la educación secundaria y de los años promedio de escolaridad, arrojan resultados robustos que confirman la importancia de inversión en capital humano en la promoción del crecimiento económico.

Las principales conclusiones a que se llega son que Colombia tiene una historia exitosa de convergencia regional, la tasa de convergencia $\beta$ se acerca al $4 \%$ anual. Las migraciones laborales no parecen desempeñar un papel importante en el proceso de convergencia, lo cual sugiere que los inmigrantes son tan productivos como los residentes. Sin duda de los hallazgos más importantes a la luz del trabajo desarrollado es que los departamentos que más invierten en educación crecen más rápido, independientemente de su posición inicial. En este sentido, el gasto público en educación resulta un elemento particularmente importante en cualquier estrategia de promoción del crecimiento.

El trabajo de Rocha y Vivas (1998), por su parte estudia los determinantes y patrones del crecimiento regional colombiano para el período 1980-1994, en el cual se analiza la efectividad de la política fiscal para corregir disparidades regionales, se aborda el tema de los efectos de la inestabilidad política y las imperfecciones del mercado sobre el crecimiento regional, y la convergencia o persistencia de las desigualdades.

De las conclusiones resulta de interés, para los fines del presente análisis, que el gasto público tiene una clara orientación redistributiva, sin que se aprecie una relación positiva con el crecimiento, las estimaciones también muestran que una parte del crecimiento del PIB se 
encuentra asociado negativamente al gasto social y que además, las transferencias regionales no contribuyen a corregir las disparidades en el PIB y contrario a los trabajos de Cárdenas, se afirma que Colombia distaba muchos de ser un caso exitoso de convergencia regional.

Bonet y Meisel (1999), en su análisis de la convergencia regional para el período 1926-1995, bajo el marco teórico de Barro y Sala- 1- Martín, y utilizando la variable depósitos bancarios departamentales como proxy del nivel de ingreso departamental, estiman la convergencia de tipo xxx, donde la empírica señala que en Colombia se dio un proceso de convergencia tipo $\beta$ entre 1926-1960, el coeficiente de correlación entre las tasa de crecimiento del período y el logaritmo del nivel inicial de los depósitos bancarios per cápita es negativo y alto (-0.89), la velocidad de convergencia para el período $1926-1950$ es de $2.5 \%$, lo que indica que el proceso es exitoso en materia de convergencia.

La convergencia tipo $\sigma$ fue calculada como la desviación estándar del logaritmo de los depósitos bancarios per cápita reales, y los resultados arrojan evidencia de un proceso exitoso de convergencia en el subperíodo al pasar de 1.25 en 1926 a 0,66 en 1960, resultado que es corroborado por el resultado del cálculo del coeficiente de variación ponderado, y el índice de Theil, el cual exhibe una tendencia a reducirse a lo largo de período, con tendencia a acercarse a cero. Cuando el índice se acerca a cero se está hablado de un sistema igualitario.

También se calculó el Índice de Concentración Herfindahl - Hirschman, mediante el cual se busca conocer la evolución de los desequilibrios regionales desde una dimensión espacial. El 
cálculo indica que en el país se dio un proceso de desconcentración espacial de los depósitos bancarios entre 1926-1960.

Entre las razones argumentadas por los autores para explicar la convergencia interdepartamental e interregional entre 1926 y 1960, está la integración vial que permitió la interconexión de las regiones y sus principales ciudades, (ferroviaria, fluvial y carreteras), y ayudó a la movilidad interdepartamental de factores, como también a incrementar la intensidad de los intercambios comerciales y la mayor integración de los mercados.

Para el período 1960-1995, se halla evidencia de una dinámica de crecimiento regional que se ajusta a los modelos de crecimiento endógeno y a la nueva geografía económica, en la cual los rendimientos crecientes a escala pueden conducir a la aglomeración espacial de la producción y a la persistencia de niveles de desarrollo desigual entre las regiones.

Las disparidades generadas en este período se atribuyen a los efectos de las políticas de fomento de la industrialización por sustitución de importaciones, la consolidación de Bogotá como gran metrópoli, y el declive económico relativo de los departamentos del Caribe.

Los resultados obtenidos en el estudio permiten concluir que Colombia tuvo un claro proceso de convergencia interdepartamental en el período 1926-1960 (tanto beta como sigma), además resulta evidente que en épocas recientes (1960-1995) la situación ha cambiado y se ha presentado una creciente polarización en los niveles de ingreso per cápita departamental. De la visión de largo plazo obtenida sobre el desarrollo departamental en Colombia en el siglo $\mathrm{XX}$, se desprende que la evolución de las disparidades interregionales depende de las 
fuerzas económicas (como las economías de escala), políticas (como las inversiones públicas) y geográficas (como los cambios en las ventajas comparativas en materia de acceso a vías de comunicación naturales). La interacción entre todas ellas es la que determina si habrá o no convergencia.

Trabajos como el de Rocha y Martínez (2003), analizan otro tipo de variables como determinantes del crecimiento regional, el trabajo explora la evidencia empírica de la conexión entre pobreza- criminalidad- crecimiento económico desde la perspectiva de la literatura de desarrollo económico, período 1981-1998. Las principales conclusiones obtenidas indican que la pobreza actúa como una condición favorable a la criminalidad y que su probabilidad puede afectar adversamente el crecimiento departamental, dado que fenómenos como el crimen y la violencia generar un clima adverso a los procesos de ahorro - inversión. El estudio afirma que el diseño de las políticas de desarrollo regional debe enfocarse a mejorar las condiciones de vida de la población, a fortalecer los procesos de formación de capital humano, la internacionalización y la gestión fiscal, lo cual permitirá apuntalar las condiciones para que las regiones se sitúen en una mayor senda de crecimiento.

Bonet( 2006) en su trabajo "Desequilibrios regionales en la política de descentralización en Colombia", concluye que la descentralización ha perpetuado las disparidades que en materia de cobertura en educación y salud existen en Colombia, anudando una gran desigualdad en los ingresos fiscales per cápita de los entes territoriales, lo cual implica que éstos asumen la responsabilidad de prestación de servicios en condiciones muy diferentes y recomienda el diseño de medidas que permitan una mayor equidad en el sistema de transferencias del 
gobierno nacional a los subnacionales, fortalecer el aparato tributario subnacional y establecer unos incentivos claros para mejorar la eficiencia del gasto.

Galvis y Meisel (2010), realizan un análisis sobre los niveles de persistencia en la pobreza de los municipios colombianos asociados a los "efectos de vecindario", con el fin de indagar si en las regiones del país operan este tipo de dinámicas; dado que en Colombia las desigualdades regionales se han mantenido y se han vuelto persistentes. Para el estudios utilizaron técnicas de la econometría espacial que permiten analizar la persistencia en la pobreza, no solo a nivel temporal, sino regional. El estudio permite concluir que cuando se efectúan las correlaciones espaciales entre las condiciones de pobreza de una municipalidad en años recientes con las de su entorno en épocas posteriores, se encuentran altas y significativas correlaciones espaciales. Ello puede ser interpretado como evidencia de la existencia de trampas espaciales, pues existen municipios que se han mantenido deprimidos, al igual que sus "vecindarios". La periferia del país enfrenta unas condiciones de pobreza estructurales a nivel regional, que la mantienen en un círculo vicioso de donde no ha sido fácil salir. También se concluye del análisis que esas condiciones de pobreza están acompañadas de inequidades tanto a nivel interregional como interpersonal. Ante esta situación de plantea la necesidad de una política más efectiva contra la pobreza con enfoque regional, que se focalice en las condiciones estructurales y de trampas de pobreza que caracterizan a la periferia colombiana. 


\section{MODELO}

\section{El modelo}

En esta sección se analiza el impacto que el gasto público en educación, salud y gasto total realizado por las entidades territoriales (32 departamentos) ha tenido sobre el crecimiento del Producto Interno Bruto Departamental para el periodo 1994 -2012.

El objetivo es dar respuesta a las preguntas a los siguientes cuestionamientos:

- ¿Han favorecido las políticas de gasto público el crecimiento económico regional?

- ¿Cuál ha sido la importancia relativa del gasto público como motor de crecimiento regional?

- ¿Qué tipo de gasto ha sido más efectivo en términos de contribución al crecimiento económico?

Con el fin de responder los interrogantes planteados se considera que existe una relación positiva entre el crecimiento del producto interno bruto - PIB departamental y el monto de los recurso públicos ejecutados. En particular, es de especial interés indagar si es el gasto en educación y salud es determinante del crecimiento o si por el contrario es el gasto total de las entidades territoriales un factor que impulse el crecimiento. En esta perspectiva se plantea la siguiente ecuación:

$$
P I B d_{i t}=c+\beta(G E d u)_{i t}+\beta(\text { GSalud })_{i t}+\beta(G T)_{i t}+\mu_{i t}
$$

Con $\mathrm{i}=1, . ., 32 \mathrm{y} \mathrm{t}=1 \ldots 18$

Donde PIBd, corresponde al producto interno bruto de cada departamento, GEdu es el gasto en educación, GSalud es el gasto en salud y Gt corresponde a los gastos totales realizados por las administraciones departamentales. 


\section{Los Datos}

Se consolidó un grupo de datos que contiene las variables seleccionadas para los 32 departamentos en el $1994-2012$.

Las series para cada una de las variables seleccionadas son las siguientes:

PIB departamental: Los datos corresponden a los publicados por el Departamento Nacional de Estadísticas DANE. La cifras originales tomadas en precios corrientes de las y empalmadas las series correspondientes a PIB- Base 1994 y PIB base 2005.

Gasto en Educación: Las cifras presentadas son las reportadas como participaciones territoriales en el sector educación y que incluyen la información de las administraciones departamentales para el periodo 1994-2012 y que son producidas por el Departamento Nacional de Planeación

Gasto en Salud: Al igual que las cifras de educación, las cifras para el gasto en salud, son las reportadas como participaciones territoriales en el sector educación y que incluyen la información de las administraciones departamentales para el periodo 1994-2012 y que son producidas por el Departamento Nacional de Planeación.

Gasto Total: El gasto total corresponde a los reportados por las administraciones departamentales como ejecución presupuestal reportada por la entidades territoriales al Departamento Nacional de Planeación.

\section{Metodología de estimación utilizada}

Considerando que el conjunto de datos materia de estudio combina series temporales (periodo 1994-2012), con unidades de sección cruzada (departamentos) se ha seleccionado la técnica de Datos Panel o datos Longitunales, que es la indicada para el estudio de estos conjuntos de datos. En un modelo econométrico de datos de panel se incluye una muestra de 
agentes económicos o de interés (individuos, empresas, bancos, ciudades, países, etc) para un período determinado de tiempo, lo que permite, combinar ambos tipos de datos (dimensión temporal y estructural).

Al aplicar la técnica de datos panel para el estudio, se busca provechar una de las principales ventajas de esta y es que permite capturar la heterogeneidad no observable, ya sea entre agentes objetos de estudio, en este caso los departamentos, así como también en el tiempo. Esta heterogeneidad no se puede detectar ni con estudios de series temporales ni tampoco con los de corte transversal.

La aplicación de esta metodología permite analizar dos aspectos de relevancia y de gran importancia y que forman parte de la heterogeneidad no observable: b) los efectos individuales específicos y ii) los efectos temporales.

Los efectos temporales son aquellos que, se induce, afectan por igual a todas las unidades individuales del estudio pero que no varían en el tiempo. Este tipo de efectos pueden asociarse, por ejemplo, a los choques macroeconómicos que pueden afectar por igual a las unidades de estudio. Por el contrario, los efectos individuales específicos hacen referencia a aquellos que afectan de manera desigual a cada uno de los individuos o agentes de estudio los cuáles son invariables en el tiempo y afectan de manera directa las decisiones que toman dichas unidades. Este tipo de efecto se identifica por lo general con la capacidad empresarial, acceso a la tecnología, eficiencia operativa, etc.

La manera como se especifican estos efectos se puede ver expresada a través de la especificación del modelo.

Un modelo de datos panel es de la forma :

$$
y_{i t}=x_{i t} \beta+u_{i t}
$$

Donde $\mathrm{i}=1, \ldots, \mathrm{N} ; \mathrm{t}=1, \ldots \mathrm{T}$ 
$X_{\text {it }}$ vector de $K$ variables explicativas (incluye una constante); $\beta$ es un vector de coeficientes y $\mu_{\text {it }}$ es el término de error que representa los efectos de todas las variables omitidas en el modelo.

El término $\mu_{\text {it }}$ se puede descomponer en:

- Los efectos no observables que difieren entre las unidades de estudio pero permanece invariable en el tiempo; generalmente asociados a la capacidad empresarial, $\mu \mathrm{i}$.

- Los efectos no cuantificables que varían en el tiempo pero no entre unidades de estudio o individuos.

Para efectos de la estimación se aplicará el método de efectos fijos que considera que existe un término constante diferente para cada individuo y supone que los efectos individuales son independientes entre sí; con este modelo se supone las variables explicativas afectan por igual a las unidades de corte transversal y que éstas se diferencian por características propias de cada una de ellas, medidas por medio del intercepto.

\section{Estimación del Modelo}

Se realiza una primera estimación del modelo planteado con datos a precios constantes 1998 y series en niveles.

\begin{tabular}{|c|c|c|c|c|}
\hline \multicolumn{5}{|c|}{ Dependent Variable: PIB } \\
\hline \multicolumn{5}{|c|}{ Method: Panel Least Squares } \\
\hline \multicolumn{5}{|c|}{ Sample: 19942012} \\
\hline \multicolumn{5}{|c|}{ Periods included: 19} \\
\hline \multicolumn{5}{|c|}{ Cross-sections included: 32} \\
\hline \multicolumn{5}{|c|}{ Total panel (balanced) observations: 608} \\
\hline \multicolumn{5}{|c|}{$\mathrm{PIB}=\mathrm{C}(1)+\mathrm{C}(2) * \mathrm{GEDU}+\mathrm{C}(3) * \mathrm{GSALUD}+\mathrm{C}(4) * \mathrm{GT}$} \\
\hline & Coefficient & Std. Error & t-Statistic & Prob. \\
\hline $\mathbf{C}(\mathbf{1})$ & 1823389. & 364774.7 & 4.998 .673 & 0.0000 \\
\hline $\mathrm{C}(2)$ & 3.872 .090 & 2.228 .655 & 1.737 .411 & 0.0000 \\
\hline $\mathbf{C}(3)$ & -5.437 .828 & 6.386 .231 & -8.514 .925 & 0.0000 \\
\hline
\end{tabular}




\begin{tabular}{|l|l|l|l|l|}
\hline C(4) & 8.575 .723 & 0.662628 & 1.294 .198 & 0.0000 \\
\hline & & & & \\
\hline R-squared & 0.975998 & Mean dependent var & 8980296. \\
\hline Adjusted R-squared & 0.973750 & S.D. dependent var & 12180381 \\
\hline S.E. of regression & 1973461. & Akaike info criterion & 3.191 .161 \\
\hline Sum squared resid & $2.16 \mathrm{E}+15$ & Schwarz criterion & 3.229 .605 \\
\hline Log likelihood & -9.648 .130 & Hannan-Quinn criter. & 3.206 .118 \\
\hline F-statistic & 4.340 .094 & Durbin-Watson stat & 0.846287 \\
\hline Prob(F-statistic) & 0.000000 & & & \\
\hline
\end{tabular}

El método de estimación es Mínimos Cuadrados Ordinarios. Debido a la dimensión temporal que tiene el panel de datos se realizaron las pruebas de estacionariedad, a nivel de grupos y de panel, para verificar la existencia de raíz unitaria o no. Los contrastes aplicados fueron : el Test de Levin - Lin (LL) (1992), el Test Im - Pesaran - Shin (IPS) (1996) y el Test de Fisher $\left(P_{\lambda}\right)(1996)$.

\begin{tabular}{|c|c|c|c|c|c|c|c|c|}
\hline \multicolumn{9}{|c|}{ Panel unit root test: Summary } \\
\hline \multicolumn{9}{|l|}{ Sample: 19942012} \\
\hline \multirow[t]{2}{*}{ Method } & \multicolumn{2}{|c|}{ Series: GEDU } & \multicolumn{2}{|c|}{ Series: GSALUD } & \multicolumn{2}{|c|}{ Series: GT } & \multicolumn{2}{|c|}{ Series: PIB } \\
\hline & Statistic & Prob. ${ }^{* *}$ & Statistic & Prob.** & Statistic & Prob.** & Statistic & Prob.** \\
\hline Levin, Lin \& Chu t* & -0.17158 & 0.4319 & -353.803 & 0.0002 & -59.668 & 0.0000 & 666.964 & 10.000 \\
\hline $\begin{array}{l}\text { Im, Pesaran and Shin W- } \\
\text { stat ** }\end{array}$ & 483.261 & 10.000 & 0.82385 & 0.7950 & -190.665 & 0.0283 & 119.670 & 10.000 \\
\hline $\begin{array}{l}\text { ADF - Fisher Chi- } \\
\text { square }^{* *}\end{array}$ & 281.757 & 10.000 & 680.715 & 0.3405 & 860.904 & 0.0342 & 316.062 & 0.9998 \\
\hline PP - Fisher Chi-square ${ }^{* *}$ & 254.433 & 10.000 & 151.849 & 0.0000 & 930.969 & 0.0102 & 934.542 & 10.000 \\
\hline \multicolumn{9}{|c|}{ *Null: Unit root (assumes common unit root process) } \\
\hline
\end{tabular}

Los resultados del p-value permiten concluir que las variables no son estacionarias por lo cual la estimación por Mínimos Cuadrado Ordinario estará dando como resultado relaciones espurias entre las variables de estudios. 
Para corregir esta situación se procedió a transformar la series pasándolas de niveles a primera diferencias de los logaritmos y nuevamente se realizó la prueba de raíz unitaria con los siguientes resultados:

\begin{tabular}{|c|c|c|c|c|c|c|c|c|}
\hline \multicolumn{9}{|c|}{ Panel unit root test: Summary } \\
\hline \multicolumn{9}{|c|}{ Sample: 19942012} \\
\hline & \multicolumn{2}{|c|}{ Series: D(GEDU1) } & \multicolumn{2}{|c|}{ Series: D(GSALUD1) } & \multicolumn{2}{|c|}{ Series: $\mathrm{D}(\mathrm{GT} 1)$} & \multicolumn{2}{|c|}{ Series: D(LPIB) } \\
\hline & Statistic & Prob.** & Statistic & Prob.** & Statistic & Prob.** & Statistic & Prob.** \\
\hline $\begin{array}{l}\text { Levin, Lin \& } \\
\text { Chu t* }\end{array}$ & -266.037 & 0.0000 & -189.056 & 0.0000 & -192.509 & 0.0000 & -162.723 & 0.0000 \\
\hline $\begin{array}{l}\text { Im, Pesaran } \\
\text { and Shin W- } \\
\text { stat }\end{array}$ & -217.613 & 0.0000 & -164.196 & 0.0000 & -163.682 & 0.0000 & -133.679 & 0.0000 \\
\hline $\begin{array}{l}\text { ADF - Fisher } \\
\text { Chi-square }\end{array}$ & 459.534 & 0.0000 & 352.059 & 0.0000 & 347.310 & 0.0000 & 290.466 & 0.0000 \\
\hline $\begin{array}{l}\text { PP - Fisher } \\
\text { Chi-square }\end{array}$ & 631.116 & 0.0000 & 412.578 & 0.0000 & 419.722 & 0.0000 & 508.804 & 0.0000 \\
\hline
\end{tabular}

Los resultados de las pruebas evidencian que la transformación de las series fue efectiva, lo cual permite realizar la estimación del panel de datos por MCO.

\begin{tabular}{|c|c|c|c|c|}
\hline \multicolumn{5}{|c|}{ Dependent Variable: LPIB1 } \\
\hline \multicolumn{5}{|c|}{ Method: Panel Least Squares } \\
\hline \multicolumn{5}{|c|}{ Sample (adjusted): 19952012} \\
\hline \multicolumn{5}{|l|}{ Periods included: 18} \\
\hline \multicolumn{5}{|c|}{ Cross-sections included: 32} \\
\hline \multicolumn{5}{|c|}{ Total panel (balanced) observations: 576} \\
\hline \multicolumn{5}{|c|}{ LPIB1 $=\mathrm{C}(1)+\mathrm{C}(2) * \mathrm{GEDU} 2+\mathrm{C}(3) * \mathrm{GSALUD2}+\mathrm{C}(4) * \mathrm{GT}$} \\
\hline & Coefficient & Std. Error & t-Statistic & Prob. \\
\hline $\mathrm{C}(\mathbf{1})$ & 0.039859 & 0.006770 & 5.887 .170 & 0.0000 \\
\hline $\mathrm{C}(2)$ & 0.000113 & 0.012945 & 0.008693 & 0.09931 \\
\hline $\mathrm{C}(3)$ & -0.140987 & 0.042195 & -3.341 .341 & 0.0009 \\
\hline $\mathrm{C}(4)$ & 2.24E-08 & $1.03 \mathrm{E}-08$ & 2.186 .260 & 0.0292 \\
\hline R-squared & 0.030756 & \multicolumn{2}{|c|}{ Mean dependent var } & 0.040357 \\
\hline Adjusted R-squared & 0.025672 & \multicolumn{2}{|c|}{ S.D. dependent var } & 0.105527 \\
\hline S.E. of regression & 0.104163 & \multicolumn{2}{|c|}{ Akaike info criterion } & -1.678 .795 \\
\hline Sum squared resid & 6.206 .189 & \multicolumn{2}{|c|}{ Schwarz criterion } & -1.648 .544 \\
\hline Log likelihood & 4.874 .929 & \multicolumn{2}{|c|}{ Hannan-Quinn criter. } & -1.666 .997 \\
\hline F-statistic & 6.050 .139 & \multicolumn{2}{|c|}{ Durbin-Watson stat } & 2.250 .678 \\
\hline Prob(F-statistic) & 0.000465 & & & \\
\hline
\end{tabular}




\section{RESULTADOS, CONCLUSIONES Y RECOMENDACIONES}

\section{Resultados}

Los resultados de la medición del PIB departamental muestran que Colombia mantiene sus resultados históricos de desigualdad regional. En el 2013 Bogotá D.C. sigue siendo la economía con mayor participación respecto al resto de departamentos con 24,7 \%, seguido de Antioquia (13,1\%), Valle del Cauca (9,3\%), Santander (7,4 \%), Meta (6,0 \%) y Cundinamarca $(5,0 \%)$, concentrando estas seis economías el 65,5\% del PIB nacional, mientras que los 10 departamentos de más baja participación en el PIB apenas suman una cifra cercana al 2\%, siendo estos Vaupés (0.03\%), Guanía (0.03\%), Vichada (0.06\%), Amazonas (0.07\%), Guaviare (0.09\%), San Andrés y Providencia (0.14\%), Chocó (0.43 \%), Caquetá $(0.45 \%)$ y Putumayo $(0.62 \%)$.

Por su parte la pobreza, vista desde los resultados del Índice de Pobreza Multidimensional se concentra de manera predominante en regiones como la Atlántica y la Pacífica con valores alrededor del 43 en 2010, situación que persiste para los años subsiguientes en los que si bien se ha presentado reducción la pobreza, siguen siendo las regiones con más pobre desempeño de sus indicadores.

\begin{tabular}{|c|c|c|c|c|c|}
\hline Regiones & 2010 & 2011 & 2012 & 2013 & 2014 \\
\hline Atlántica & 45,5 & 41,8 & 41,1 & 37,4 & 34,6 \\
\hline Oriental & 28,6 & 29,2 & 28,5 & 24,8 & 18,0 \\
\hline Central & 31,2 & 30,7 & 26,7 & 26,1 & 28,1 \\
\hline Pacífica (sin incluir Valle) & 42,7 & 41,4 & 36,3 & 37,6 & 34,6 \\
\hline Bogotá & 12,1 & 11,9 & 11,1 & 8,7 & 5,4 \\
\hline Valle del Cauca & 24,1 & 24,7 & 20,5 & 16,2 & 15,7 \\
\hline Antioquia & 27,8 & 25,7 & 21,7 & 22,4 & 19,5 \\
\hline
\end{tabular}

Fuente: DANE - Encuesta Nacional de Calidad de Vida.

De otra parte, entre las regiones que muestran mejores indicadores se encuentra Bogotá, Antioquia, Valle, y la región oriental que coinciden con los departamentos de mas altas tasas de crecimiento de su PIB. 
La condiciones socioecnómicas derivadas de esta situación de disparidad regional se ven reflejadas en los resultados de calidad de vida de los habitantes, en indicadores como tasas de mortalidad infantil los departamentos que están por debajo de la media nacional (17.78\%) son Valle del Cauca (12.91\%), N. de Santander (13.2\%), Caldas (13.35\%), Risaralda (13.68\%), Cundinamarca (13.88\%), Santander (14.13\%), Antioquia (15.05\%), San Andrés y Providencia (15.33\%), Boyacá (16.04\%) y Atlántico (16.63\%). De otra parte, manteniendo el patrón sistemático los departamentos con los resultados más críticos son La Guajira (32.05\%), Guaviare (35.05), Vichada (36.08), Guainía (36.71\%), Vaupés (41.91\%), Chocó (42.69\%) y Amazonas (46.44\%).

Estos datos además son consistentes con los resultados de desempeño fiscal ${ }^{10}$. Para la vigencia 2013 las finanzas de los gobiernos municipales y departamentales cerraron la vigencia 2013 con un saldo positivo, al registrarse gastos por más de $\$ 76,9$ billones e ingresos por $\$ 79,1$ billones, lo que equivale a un superavit de $0,77 \%$ del Producto Interno Bruto -PIBy equivalente a $\$ 2,1$ billones. De los resultados de la medición se evidencia como solo Bogotá y otras cuarenta ciudades concentran cerca del $80 \%$ de las finanzas municipales, mientras que seis departamentos recogen cerca del $70 \%$ de las finanzas departamentales; entre los que se destacan Cundinamarca (77,9 puntos), Sucre (77,5 puntos) y Cesar (77 puntos) como los departamentos con los desempeños más robustos. Entre tanto, el resultado crítico en el Desempeño Fiscal departamental, sigue siendo para los departamentos de Chocó, San Andrés y Putumayo con 54,7, 52,4 y 49, 6 puntos respectivamente.

\footnotetext{
${ }^{10}$ Desempeño fiscal de los departamentos y municipios 2013. Informe del Departamento Nacional de
} Planeación (Artículo 79 Ley 617 de 2000). ISSN 2027-5838 
En este punto es importante resaltar la dependencia de las entidades territoriales de las transferencias de la Nación y las Regalías (SGR). De acuerdo con el Informe de desempeño fiscal de los departamentos 2013, los recursos de Transferencias y Regalías tiene una participación en el total de los ingresos de los departamentos cercana al $65 \%$.

Tabla 1. Ingresos Totales de los Gobiernos Departamentales -2013.

\begin{tabular}{|lccc|} 
Cuenta & \% del PIB & $\begin{array}{c}\text { Miles de millones } \\
\text { de pesos }\end{array}$ & $\begin{array}{c}\text { Participación } \\
\text { \% en Ingresos }\end{array}$ \\
\hline Ingresos Totales & $\mathbf{3 , 1 4}$ & $\mathbf{2 6 . 6 6 3}$ & $\mathbf{1 0 0 , 0 0}$ \\
Ingresos Corrientes & $\mathbf{1 , 0 4}$ & $\mathbf{7 . 2 3 4}$ & $\mathbf{2 7 , 1 3}$ \\
Tributarios & 0,79 & 5.720 & 21,46 \\
No tributarios & 0,21 & 1.383 & 5,19 \\
Transferencias Corrientes & 0,04 & 204 & 0,77 \\
Ingresos de Capital & $\mathbf{2 , 1}$ & $\mathbf{1 9 . 4 2 8}$ & $\mathbf{7 2 , 8 7}$ \\
Transferencias & 1,48 & 10.686 & 40,08 \\
Cofinanciación & 0,04 & 584 & 2,19 \\
Regalías & 0,36 & 6.648 & 24,93 \\
Otros & 0,22 & 1.511 & 5,67 \\
\hline Fuente: Cálculos -DNP-DDTS, con base en información reportada por las entidades territoriales a través del \\
Formulario Único Territorial - FUT- 2013.
\end{tabular}

Ahora bien, en términos de cobertura de los sistema de educación y salud, las condiciones de desigualdad se mantienen: Para el caso de la educación, según Fedesarrollo (2009) “al analizar la evolución de la cobertura a nivel departamental (sin incluir a los distritos (salvo Bogotá D.C.) y a los municipios certificados) se observa que hay grandes desigualdades en las tasas de cobertura bruta entre los entes territoriales y que, al igual que a nivel nacional, existen deficiencias importantes en los niveles de transición, básica secundaria y educación media (en primaria únicamente salta a la vista la baja cobertura bruta en San Andrés y Providencia, que no alcanza el 80\%; ver gráficos 21-24).

En particular, se destaca que los Antiguos Territorios Nacionales tienden a presentar bajas tasas de cobertura. A pesar de que se han observado grandes avances en la tasa de cobertura bruta en primaria en estos departamentos, algunos de estos (Vichada, Guaviare, y Vaupés) 
tienen tasas de cobertura bruta inferiores al 65\% en transición (frente a 94\% a nivel nacional). Así mismo, Vaupés y Vichada se encuentran por debajo del $60 \%$ en básica secundaria (frente a $98 \%$ a nivel nacional), y de manera aún más preocupante, Guaviare, Vaupés y Guainía no alcanzan el $40 \%$ en tasa de cobertura bruta en educación media (frente a 71,3\% a nivel nacional). Chocó es otro departamento que se destaca por sus bajas tasas de cobertura bruta en todos los niveles. El análisis de las coberturas netas también corrobora las profundas desigualdades que existen entre los departamentos. Por ejemplo, para el caso de transición, las tasas de cobertura oscilan entre $23,7 \%$ en Vaupés y 77,1\% en Cundinamarca.

Las diferencias en cobertura neta en transición claramente están asociadas, en parte, con aspectos culturales; por ejemplo, es un hecho que los niños y niñas en áreas rurales suelen comenzar el ciclo educativo a una edad más tardía que sus contrapartes urbano. Este fenómeno también se asocia con las altas desigualdades en la tasa de cobertura neta en la educación media, donde Guainía presenta una tasa de tan sólo 7,5\%, mientras que Bogotá alcanza un nivel de 50,8\%."

En términos de cobertura en salud, ya sea bajo el régimen contributivo o subsidiado, de acuerdo con los datos del Ministerio de Salud 2012, se alcanza una tasa del 91\%. Aun cuando las disparidades en este aspecto no son tan pronunciadas, las tasas por debajo del $80 \%$ corresponde a los departamentos de Vaupés, Amazonas, Guaviare, San Andrés y Cundinamarca con valores del 58\%, 69\%, 70\%, 74\% y $77 \%$ respectivamente.

Una vez que se han relacionado algunas resultados relevantes para comprender la dinámica del desempeño departamental, el resultado de las estimaciones econométricas. El modelo 
estimado permite inferir que la dinámica del gasto público del orden nacional se cumple en las regiones y la relación entre el crecimiento del PIB departamental y el gasto público es positiva, por tanto y en concordancia con el modelo teórico planteado, el gasto público departamental genera dinámicas positivas que permiten apalancar el crecimiento económico y por ende las condiciones de vida de la población.

Visto de manera individual, por cada tipología de gasto que se usó para la estimación los resultados muestran la relación esperada entre el gasto en educación y el gasto total con el crecimiento del PIB departamental; no así con el gasto en salud, aun cuando los estadísticos de prueba no son los suficientemente robustos para concluir que el este tipo de gasto resulte inefectivo en término del crecimiento económico y calidad de vida.

Una de las razones por las que pueden explicar este resultado esta relaciona con el monto total de recurso destinado por cada departamento al cada sector, en la que de manera sistemática por $\$ 1$ peso que se destina al sector salud se están destinando $\$ 2.5$ al sector educación. Adicionalmente, los resultados en términos de cobertura muestran que mientras en salud se han alcanzado tasas por encima del $80 \%$ en la mayoría de los territorios, en educación persisten las disparidades regionales.

Por último, con respecto a la robustez de los resultados es importante mencionar la precariedad de la producción estadística regional, que no permiten contar con series 
unificadas en su metodología y periodicidad que permitan garantizar la efectividad de los resultados de las estimaciones.

\section{Conclusiones y Recomendaciones.}

1. En Colombia existen y persisten desigualdades regionales que condicionan un desarrollo y una garantía de derecho inequitativos para la población.

2. El sector público juega un rol fundamental para garantizar los preceptos del estado social de derecho que garantice la vida digna y contrarreste las desigualdades sociales existentes en aras de ofrecer a todos las oportunidades necesarias para desarrollar sus capacidades.

3. El gobierno nacional debe trabajar en pro del mejoramiento del desempeño de las entidades territoriales que les permita perfeccionar su capacidad gerencial, mejorar su cultura institucional, fortalecer la planeación y ejecución de políticas, programas, planes y proyectos que propendan por la implementación de esquemas gestión para resultados de desarrollo (GpRD), en la cual la información del desempeño se usa para mejorar la toma de decisiones y a través de herramientas prácticas para la planificación estratégica, la gestión de riesgos, el monitoreo del progreso y la evaluación de los resultados se logra el desarrollo integral del territorio y superación de los altos niveles de pobreza y desigualdad social de la población. 
4. Sigue siendo un reto el diseño de política que reduzca las desigualdades en el crecimiento económico y permita superar los indicadores de pobreza. Al respecto, resultará de especial interés evaluar el impacto del uso de los instrumentos recientes tales como el Sistema General de Regalías, las alianzas público privadas, los Contratos Plan, así como las diversas formas de asociación previstas en la Ley Orgánica de Ordenamiento Territorial.

5. A futuros trabajos y con mejores estadísticas disponibles sería importante indagar si el peso de los fenómenos de corrupción contribuyen de manera diferenciada a los resultados de desempeño económico y de condiciones de vida, o si por el contrario afecta de manera indistinta a las regiones y niveles de gobierno. 


\section{LISTA DE REFERENCIAS}

Aschauer, D. (1989c), "Does Public Capital Crowd out Private Capital", Journal of Monetary Economics, 24: 171-188.

Barón, J. D. (2003). “¿Qué sucedió con las disparidades económicas regionales en Colombia entre 1980 y el 2000?”, Documentos de trabajo sobre Economía Regional 38, Centro de Estudios Económicos Regionales, Banco de la República, Cartagena.

Barón, Juan David y Adolfo Meisel (2003). "La descentralización y las disparidades económicas regionales en Colombia en la década de 1990", Documentos de trabajo sobre economía regional 36, Cartagena: Banco de la República, Centro de Estudios Económicos Regionales (CEER).

Barro, R. (1990), "Government Spending in a Simple Model of Economic Growth", Journal of Political Economy, 98, S103-S125. Barro, R. (1991), "Economic Growth in a Cross-Section of Countries", Quarterly Journal of Economics, 106: 407-444

Barro, R., "Economic Growth in a Cross Section of Countries", Quarterly Journal of Economics, Vol. 2, No. 106, 1991.

Barro, R., "Economic Growth in a Cross Section of Countries", Quarterly Journal of Economics, Vol. 2, No. 106, 1991.

Barro, R., Economic Growth, McGraw-Hill, New York, 1995.

Barro R. \& X. Sala-i-Martin "Economic Growth and Convergence Across the United States", NBER Working Papers, No. 3419.

Barro R. \& X. Sala-i-Martin, (1995), Economic Growth, McGraw-Hill.

Bonet, Jaime y Adolfo Meisel (1999). "La convergencia regional en Colombia: una visión de largo plazo 1926- 1995”, Coyuntura Económica, V.XXIX. Bogotá: Fedesarrollo, 1999.

Bonet.J.m "Desequilibrios regionales en la política de descentralización en Colombia”.Documentos de trabajo sobre Economía Regional. N 77. Banco de la República. 2006.

Cárdenas,M. “Crecimiento y Convergencia en Colombia: 1950-1990”. Revista Planeación y Desarrollo. Edición Especial - DNP 35 Años. 1993.

Cárdenas,M., Pontón,A., y Trujillo,Juan. "Convergencia y Migraciones Inter-departamentales en Colombia: 1950-1989”. Revista Coyuntura Económica.Vol 23. № 1, 1993.

Cárdenas, M., \& Escobar, A., "Infraestructura y crecimiento departamental 1950-1994”, Planeación y Desarrollo vol. XXVI (4), Bogotá: DNP,1995.

Gujarati, Damodar N.’Econpometría” McGraw Hill. Cuarta Edición. 2007.

Dasgupta, D., "Government Spending in a Model of Endogenous Growth with Private and Public Capital”. Indian Statistical Institute. 2001. 
Departamento Nacional de Planeación DNP, Índice de Pobreza Multidimensional (IPM-Colombia) 1997-2008 y meta del PND para 2014. 2011.

Doménech, R., "Política Fiscal y Crecimiento Económico". Universidad de Valencia. 2004

Galvis, L. \& A. Meisel (2000). "El crecimiento económico de las ciudades colombianas y sus determinantes, 1973-1998". Meisel, A. (ed.). Regiones, ciudades y crecimiento económico en Colombia, Bogotá: Colección de Economía Regional, Banco de la República. http://www.banrep.gov.co/sites/default/files/publicaciones/archivos/DTSER18Crecimientociudades.pdf

Galvis, L. \& A. Meisel. "Persistencia de las desigualdades regionales en Colombia: Un análisis espacial". Documentos de Trabajo sobre Economía Regional. № 120. Banco de la República. Bogotá.2010.

Mayorga, M., Muñoz, E., "La técnica de datos de panel una guía para su uso e interpretación". Documento de trabajo. División Económica, Departamento de Investigaciones Económicas. Banco Central de Costa Rica.2000.

http://www.seti.chubut.gov.ar/Publico/PDF/Mayorga\%20y\%20Mu\%C3\%B1oz\%20Tecnica \%20de\%20datos\%20de\%20panel.pdf

McGrattan, E., “A Defense of AK Growth Models” Quartely Review. Vol 22, N 4. Federak Reserve Bank Minneapolis Quarterly Review. 1998.

Pindyck, Robert y Rubinfeld, Daniel. 1993. Econometric Models \& Economic Forecasts.

McGraw-Hill International Editions, Fourth Edition.

Posada, C., \& Gómez , W. “ Crecimiento económico y gasto público: un modelo para el caso colombiano” Borradores de Economía N²18. Banco República. 2002.

Posada, C., \& Escobar, J. " Crecimiento económico y gasto público: una interpretación de las experiencias internacionales y del caso colombiano (1982-1999)”. Borradores de Economía N²58. Banco República. 2003.

Rocha,R. \& Martinez,H. "Pobreza, crimen y crecimiento regional en Colombia". Archivos de Economía. Documento 230, 2003.

Rocha, R. \& A. Vivas (1998). “Crecimiento regional en Colombia: ¿persiste la desigualdad?”, Revista de Economía del Rosario, 1, 1. 1998.

Romero, D., Strauch R,. "Public Finances And Long - Term Growth in Europe - Evidence From A Panel Data Analysis". Working Paper Series. European Central Bank. 2003

Sánchez, Fabio., "El papel del capital público en la producción, la inversión y el crecimiento económico en Colombia. Archivos de Macroeconómia. Documento 18. Departamento Nacional de Planeación. 1993.

Santa María, M., Millán, N., Moreno, J., \& Reyes, C., "LA DESCENTRALIZACIÓN Y EL FINANCIAMIENTO DE LA SALUD Y LA EDUCACIÓN EN LOS DEPARTAMENTOS: 
¿CUÁLES SON LAS ALTERNATIVAS?”. Informe presentado a la Federación de Municipios. Capítulo Educación. Fedesarrollo. 2009. 